\title{
Philosophiques
}

\section{Pour une gnoséologie objective et applicable}

\section{Éric Bourneuf}

Volume 23, numéro 2, automne 1996

URI : https://id.erudit.org/iderudit/027396ar

DOI : https://doi.org/10.7202/027396ar

Aller au sommaire du numéro

Éditeur(s)

Société de philosophie du Québec

ISSN

0316-2923 (imprimé)

1492-1391 (numérique)

Découvrir la revue

Citer cet article

Bourneuf, É. (1996). Pour une gnoséologie objective et applicable. Philosophiques, 23(2), 265-283. https://doi.org/10.7202/027396ar

\section{Résumé de l'article}

Je présente les grandes lignes d'une approche méthodologique des questions gnoséologiques traditionnelles, qui intègre mais dépasse l'approche habituelle consistant à construire des théories pour rendre compte des intuitions particulières des locuteurs eu égard à l'obtention de conditions épistémiques. Mon analyse, qui se situe donc au niveau méta-gnoséologique, m'amène à définir mon propre projet gnoséologique par renonciation de deux nouvelles contraintes sur les théories gnoséologiques : l'applicabilité et l'objectivité.
Ce document est protégé par la loi sur le droit d'auteur. L'utilisation des services d’Érudit (y compris la reproduction) est assujettie à sa politique d'utilisation que vous pouvez consulter en ligne.

https://apropos.erudit.org/fr/usagers/politique-dutilisation/ 


\title{
POUR UNE gNOSÉ0LOGIE OBJECIIVE EI APPLICABLE
}

\author{
PAR \\ Éric Bourne UF
}

\begin{abstract}
RÉSUMÉ: Je présente les grandes lignes d'une approche méthodologique des questions gnoséologiques traditionnelles, qui intègre mais dépasse l'approche habituelle consistant à construire des théories pour rendre compte des intuitions particulières des locuteurs eu égard à l'obtention de conditions épistémiques. Mon analyse, qui se situe donc au niveau métagnoséologique, m'amène à définir mon propre projet gnoséologique par l'énonciation de deux nouvelles contraintes sur les théories gnoséologiques : l'applicabilité et l'objectivité.
\end{abstract}

ABSTRACT:I outline my view of a top-down methodological approach to traditional epistemological questions, and contrast it with the bottom-up approach, more common in contemporary literature, oriented towards the rationalization of specific epistemic intuitions. My analysis, located at the metaepistemological level, leads me to a new conception of traditional epistemology, through the identification of two extra constraints on epistemological theories, besides the appeal to intuitions : applicability and objectivity.

\section{Un éventail d'approches et de théories gnoséologiques}

Le lecteur le moindrement assidu de gnoséologie ${ }^{1}$ anglo-saxonne contemporaine ne pourra manquer de constater la grande diversité des positions philosophiques qui s'y retrouve. De façon générale, on peut diviser les participants aux débats en deux grands groupes de protagonistes, les traditionalistes et les naturalistes, qui ne s'entendent pas sur la nature, les buts et la méthodologie de l'entreprise gnoséologique, mais qui s'opposent tous deux à un petit groupe de

1. C'est le terme que j'utiliserai comme traduction de l'expression anglaise "epistemology", faute de mieux, pour éviter les malentendus qui pourraient ètre suscitès par une traduction littérale, étant donné qu'" êpistémologie " signifie généralement "philosophie des sciences" en français. La gnoséologie est donc pour moi la discipline philosophique concernée par l'étude des conditions de validité des énoncés et des groupes d'énoncés factuels (ou encore, ce qui n'est pas nécessairement equivalent, des énoncés et des groupes d'énoncés ayant une valeur de vérité). Certains prêtendront reconnaitre ici la théorie de la connaissance, mais j'ai tendance à penser que cette dermière n'en désigne qu'une conception particulière. En effet, certaines version de l'epistemology ne sont pas à proprement parler des theories of knowledge puisqu'elles ne considèrent pas les énoncés factuels en tant qu'énoncés de connaissance (comme par exemple la théorie de van Fraassen - voir la note 11 ). 
pragmatistes, qui proclament à grands cris que la gnoséologie a fait son temps. La bataille principale entre les deux premiers rivaux concerne la question du statut de la gnoséologie par rapport à la science, celle de savoir si la première peut exister en tant que discipline autonome ayant autorité sur la seconde, comme l'affirment les traditionalistes, ou si elle n'en est qu'un chapitre, comme le prétendent les naturalistes. Là où les traditionalistes se représentent la gnoséologie en tant qu'analyse purement aprioriste de concepts épistémiques, applicables notamment aux théories et aux énoncés scientifiques, les naturalistes veulent surtout voir une étude empirique, scientifique, des conditions de production et de validation de la connaissance. Les pragmatistes, quant à eux, objectent notamment que toute gnoséologie traditionnelle repose sur le concept vicié de vérité-correspondance, ce qui annule pratiquement l'espoir de formuler une théorie cohérente de ce type ${ }^{2}$.

Se représenter la scène des discussions contemporaines sur la gnoséologie en trois camps belligérants bien circonscrits ne rend cependant pas compte de l'ampleur des divergences qui existent entre les acteurs impliqués. D'abord, tous les naturalistes ne s'accordent pas entre eux sur l'attitude à adopter par rapport à la gnoséologie traditionnelle. Selon Susan Haack, on peut distinguer des naturalistes révolutionnaristes, qui déclarent la discipline traditionnelle illégitime ou mal conçue, des naturalistes réformistes, pour qui l'analyse des concepts épistémiques demeure essentielle mais doit utiliser les résultats de la science cognitive, et des naturalistes expansionnistes, qui veulent joindre les démarches de la science cognitive et de la gnoséologie traditionnelle dans un projet commun ${ }^{3}$. Par ailleurs, les traditionalistes ne forment pas non plus un groupe unifiè. Certains d'entre eux considèrent que le but de leurs analyses conceptuelles est l'élaboration de critères épistémiques qui rendraient compte de nos pratiques courantes, dautres visent la légitimation ou la réforme de ces pratiques épistémiques, tandis que plusieurs sont avant tout concernés par la réfutation $\mathrm{du}$ scepticisme (ces tâches ne sont pas nécessairement exclusives) ${ }^{4}$.

L'ensemble des débats mentionnés précédemment constitue la dimension méta-gnoséologique du problème : ils touchent la question de définir la nature, le but et la méthodologie de la gnoséologie en tant que discipline philosophique. Au niveau proprement gnoséologique, celui des théories particulières qui s'inscrivent dans un cadre méta-gnoséologique donné, le degré de consensus n'est pas tellement plus grand, car mème lorsqu'il y a entente sur la bonne façon de concevoir la gnoséologie, celle-ci n'est souvent que

2. Sur ce que j'appelle ici le pragmatisme, voir, par exemple, Richard Rorty, Philosophy and the Mirror of Nature, Princeton, Princeton University Press, 1979, et Steven Stich. The Fragmentation of Reason, Cambridge, MIT Press, 1990 .

3. Voir Susan Haack, Evidence and Inquiry. Oxford, Basil Blackwell, 1993. chap. 6.

4. Les diffèrences d'approche au sein des traditionalistes auxquelles je réfère ici sont discutees notamment par Laurence Bonjour dans The Structure of Empirical Knowledge, Cambridge, Harvard University Press, 1985, p. 8-15, et Alan Millar dans Reasons and Experience, Oxford, Clarendon Press, 1991, chap. 7. 
superficielle. Par exemple, tous les naturalistes révolutionnaristes ne partagent pas une même vision de la psychologie cognitive, tandis que les traditionalistes qui reconnaissent la fonction revisionniste de l'analyse conceptuelle peuvent s'opposer par ailleurs sur la question de savoir si le concept épistémique central est celui de connaissance, de justification ou de rationalité, pour ne nommer que les principaux, ainsi que sur la signification à donner à ces notions.

Que conclure de toute cette variété ? Que la gnoséologie est en excellente santé, puisqu'elle manifeste tous les signes d'une discipline philosophique en pleine effervescence ou, au contraire, qu'elle est sans orientation précise ni fondation stable, comme la science dans une période pré-paradigmatique? Ici, il ne faut pas perdre de vue que nous parlons d'une discipline normative, même sous sa version naturaliste ${ }^{5}$, et que louanger la diversité risque d'équivaloir, dans ce contexte, à se commettre envers une forme de relativisme. Je me dois d'indiquer mon parti pris immédiatement, pour ètre honnète envers le lecteur, même si je ne peux me justifier dès maintenant: je retiendrai comme hypothèse de départ l'idée que le relativisme n'est pas ultimement une solution acceptable en gnoséologie. Par conséquent, il faudra trouver une autre explication à la situation qui prévaut actuellement dans le domaine et chercher à y réduire la prolifération de théories contradictoires. Cet article est consacré à la réalisation de ce double objectif.

Dans la section suivante, j'amorce la discussion en fonction du second objectif, en partant à la recherche de contraintes méthodologiques que l'on pourrait imposer aux théories gnoséologiques, de façon à restreindre le champ de leur acceptabilité. Puisqu'il m'est impossible dans le cadre d'un article aussi court de faire le point sur toutes les questions soulevées en introduction, je me situerai d'entrée de jeu au sein de la gnoséologie traditionnelle, réservant de ce fait mes réflexions sur le naturalisme et le pragmatisme pour une autre occasion. J'aurai quand mème l'occasion de commenter une approche théorique privilégiée par des naturalistes réformistes, le fiabilisme, qui ne rompt pas avec le traditionalisme de façon aussi radicale que celles de leurs homologues révolutionnaristes.

\section{La nécessité de contraintes méthodologiques supplémentaires}

On retrouve, au sein de la gnoséologie traditionnelle, un certain niveau de base d'accord tacite partagé par la plupart des participants aux débats, qui résiste à toute la diversité que je viens d'évoquer. En effet, une grande majorité d'entre eux s'accordent pour dire qu'il faut parvenir à formuler des concepts épistémiques permettant à la fois la clarification et la systématisation de nos concepts épistémiques courants. La référence aux concepts épistémiques courants, qu'exprime surtout l'exigence de clarification, s'explique par le fait que l'on tient à ce que les concepts de la gnoséologie préservent nos intuitions dans des cas évidents (comme, par exemple, celui qui consiste à

5. Mème Guine, qui peut, selon une certaine interprétation, ètre considéré comme un naturaliste révolutionnariste, se fixe un objectif normatif. Voir Pursuit of Truth Cambridge, Harvard University Press, 1992, p. 19. 
reconnaitre la rationalité des énoncés à propos de la réalité imperceptible à l'œil nu obtenus par l'intermédiaire d'observations télescopiques). La gnoséologie traditionnelle ne doit pas s'éloigner du sens commun à un point tel qu'elle perde le contact avec l'origine de sa motivation première, lidentification des conditions de validité de nos croyances et de nos théories, qui informe déjà nos catégories évaluatives courantes. Quant à l'exigence de systématisation, elle tient au fait que les concepts épistémiques courants sont souvent vagues ou sujets à interprétation, et donc peu appropriés pour l'élucidation de cas plus difficiles, qui font également partie de l'objectif de la démarche gnoséologique. Dans de tels cas, comme par exemple celui qui touche la question de notre connaissance d'un réel indépendant, les intuitions sont divergentes justement parce que les concepts courants sont trop flexibles.

Le point de départ que constitue la rationalisation d'intuitions courantes semble donc ètre un élément incontournable de toute validation d'une théorie gnoséologique. Cependant, dans la mesure où ils convoitent l'espoir d'en arriver un jour à une forme d'accord au sein de leur discipline, les gnoséologues doivent commencer par reconnaitre que ce n'est pas du tout un critère d'acceptabilité suffisant. La démonstration de cette affirmation est facile. D'abord, il devrait aller de soi que les intuitions en question sont d'ordre sémantique. Par exemple, l'intuition que telle situation représente une occurrence de connaissance et l'intuition que notre concept de connaissance s'y applique sont identiques. Ensuite, il est évident que tous les gnoséologues n'emploient pas nécessairement les mémes concepts épistemiques. Dans ce contexte, il est permis d'imaginer la situation suivante, certes possible: un gnoséologue propose un certain concept épistémique comme systématisation d'un concept courant; à mesure qu'il se familiarise avec ses ramifications, en le confrontant avec les situations-test considérées dans la littérature, ce concept devient opérationnel chez lui, si bien que ses intuitions quant au statut épistémique de nos croyances sont désormais simplement celles qu'il s'applique. Il suffit maintenant d'imaginer que ce cas ne soit pas isolé pour déboucher sur une bataille d'intuitions sans résolution possible. Conclusion: la méthode gnoséologique qui repose prioritairement sur les intuitions des locuteurs n'est pas adéquate pour trancher la question ${ }^{6}$.

Il est vrai qu'il existe dans la littérature un certain nombre de contraintes méthodologiques supplémentaires dont l'imposition aux théories gnoséologiques est considérée. Les plus immédiates concernent différentes formes de cohérence, telles que la cohérence logique, la cohérence explicative et l'auto-référence (lorsqu'une théorie gnoséologique valide ses propres principes). On invoque aussi fréquemment des vertus pragmatiques comme la simplicité et le contenu informatif. L'utilité et la valeur de ces exigences, qui ne sont pas triviales, et qui permettent parfois l'élimination de certaines théories gnoséologiques, doivent ètre reconnues. La contrainte de

6. Cet argument s'inspire du fondationalisme sémantique de Paul Moser, la position selon laquelle les fondements de la gnoséologie traditionnelle sont sémantiques. Voir Philosophy After Objectivity. Oxford, Oxford University Press, 1993, chap. 2 et 3 . 
simplicité me semble particulièrement importante et trop souvent négligée. Je ne suis d'ailleurs pas convaincu qu'il soit juste de la qualifier seulement de pragmatique: comment, en effet, pouvonsnous expliquer le fait qu'il soit relativement aisé pour plusieurs, même lorsqu'ils ne sont pas gnoséologues au départ, de se prononcer sur l'application des concepts épistémiques dans les situations complexes et artificielles qui servent de test dans la littérature, s'ils n'emploient pas des concepts assez simples ? Quoi qu'il en soit, il est cependant clair, à mon avis, que des discours épistémiques à la fois cohérents et pratiques peuvent ètre incompatibles. La situation actuelle l'atteste : les contraintes habituelles sont insuffisantes.

Je me propose donc, dans ce texte, de considérer deux nouvelles contraintes à imposer aux théories gnoséologiques, l'objectivité et l'applicabilité, et d'illustrer leur apport méthodologique en montrant comment elles permettent de rejeter certaines théories gnoséologiques insatisfaisantes à leur égard. La compréhension de la nature de ces contraintes requiert l'exposition des bases de ma propre version de la gnoséologie traditionnelle, baptisée "gnoséologie cartésienne ", à laquelle je vais maintenant procéder.

\section{Gnoséologie pure el gnoséologie appliquée}

En un mot, la gnoséologie cartésienne est selon moi la thèse que la gnoséologie a pour but de donner des règles de conduites objectives à un niveau très général aux sujets épistémiques qui font l'évaluation du statut de leurs opinions dans le but d'acquérir un système étendu de croyances vraies. Elle est aussi, comme je viens de le mentionner, ma version de la gnoséologie traditionnelle, mais ceci ne signifie pourtant pas que tous les travaux regroupés sous cette dernière étiquette peuvent ètre qualifiés de "cartésiens". En fait, la plupart ne le peuvent pas, à mon avis, puisqu'un aspect essentiel du projet de Descartes y est notablement absent. La gnoséologie traditionnelle, telle qu'elle se pratique en cette seconde moitié du vingtième siècle, est généralement concernée par l'analyse de concepts épistémiques tels que connaissance, justification et rationalité. L'objectif de Descartes, cependant, ne se situait pas réellement ou pas exclusivement sur ce plan, mais était plutōt celui de mettre de l'avant une méthode qui pouvait permettre à un sujet d'effectuer une évaluation objective de ses opinions, dans le but de parvenir à la connaissance des objets accessibles à l'intelligence humaine. C'est la référence au sujet épistémique partant à la recherche d'une méthode utilisable qui explique la caractérisation de mon projet gnoséologique en tant que cartésien ${ }^{7}$, et non pas mon adhésion à la propre solution de Descartes à ce problème, qui propose au sujet d'identifier des idées claires et distinctes en lesquelles il peut croire avec certitude.

La gnoséologie cartésienne n'est donc pas seulement l'analyse des conditions sous lesquelles un certain statut épistémique se réalise chez un sujet, mais aussi celle des conditions sous lesquelles un

7. Je m'inspire ici de la caractérisation de la gnoséologie cartésienne donnée par Richard Foley dans Working Without a Net, Oxford, Oxford University Press, 1993, et plus particulièrement p. $117-131$. 
sujet peut prétendre réaliser ce statut épistémique. Pour bien se représenter la nature de la distinction que je viens d'introduire, on peut dire qu'elle est l'équivalent, au niveau des notions épistémiques, de celle qui existe dans le cas du concept de vérité, entre la nature et les critères de la véritè. C'est en effet une chose de savoir en quoi consiste la véritè, mais cela, en soi, ne nous dit pas comment l'atteindre ; de la mème façon, savoir ce qu'est la justification ou la rationalité n'équivaut pas à savoir lesquelles de nos opinions sont justifiées ou rationnelles. Il y a donc deux projets distincts au sein de la gnoséologie cartésienne, que je nomme respectivement "gnoséologie pure " et "gnoséologie appliquée ".

Le caractère distinctif des deux projets gnoséologiques que je mets en évidence apparait le plus facilement en contrastant les différentes perspectives qu'elles adoptent. D'une part, la gnoséologie pure considère les problèmes gnoséologiques d'un point de vue absolu, comme s'il était possible pour ses théoriciens de s'extraire de la condition humaine afin d'y porter un regard omniscient. Son objectif premier est de spécifier conceptuellement les conditions constitutives de réalisation de certains statuts épistémiques ${ }^{8}$. Ainsi, la gnoséologie pure n'est concernée par aucune forme d'évaluation, et ce fait est manifeste dans sa méthode: lorsqu'il faut se demander, par exemple, si un sujet sait ou non dans une situation inhabituelle du type que l'on retrouve fréquemment dans la littérature sur ce qu'il est maintenant convenu d'appeler "le problème de Gettier ", toute l'information pertinente est donnée dans la description de l'exemple. Le théoricien considère des situations possibles, hypothétiques, qu'il a souvent lui-même définies; il ne cherche pas à se placer dans la peau de son sujet pour savoir si celui-ci pense ou non savoir de son point de vue. Ce type de situation réelle, dans laquelle le sujet épistémique doit faire sa propre analyse des faits probants (evidence) disponibles, est plutôt l'objet d'étude de la gnoséologie appliquée. En effet, dans la vie courante, un sujet ne peut s'extraire de sa propre perspective pour accéder à la vérité absolue sur une question, telle qu'elle serait perceptible par un observateur omniscient. Les faits probants ne se donnent généralement pas à sa conscience directement, comme s'ils étaient déposés sur la surface des choses ; s'enquérir de leur présence requiert l'exercice de la capacité de juger, d'évaluer, de faire des estimations, avec tous les risques d'erreur que cela comporte. La gnoséologie appliquée aborde donc les problèmes gnoséologiques selon la perspective réelle du sujet: son objet d'étude principal, qui la caractérise en propre, est le jugement épistémique. L'adjectif "appliqué " ne doit pas nous faire croire à tort qu'elle consiste en l'application à des problèmes précis, comme la connaissance perceptuelle ou la connaissance du passé, de résultats généraux obtenus par la gnoséologie pure, puisqu'elle est avant tout une gnoséologie utilisable pour le sujet, dans la gestion de ses opinions.

8. Il ne faut donc pas critiquer ici la gnoséologie pure, sous prétexte qu'elle adopte un point de vue absolu ou omniscient, en faisant valoir l'impossibilité de la chose. Ce serait perdre de vue que sa tâche est purement conceptuelle. 
Il me paraît exact de dire que la gnoséologie pure et la gnoséologie appliquee sont toutes deux concernées par l'examen des relations entre croyance, fait probant et vérité, bien que la vérité ne soit souvent qu'un idéal régulateur dans le cadre de la seconde. Par contre, les notions de croyance et de fait probant doivent ètre conçues de façon différente selon le projet. Une distinction entre croyance et opinion peut être introduite pour marquer un premier contraste important. Je définis, d'une part, le concept d'opinion comme lacceptation consciente et réfléchie d'un énoncé, sur la base d'un ou de plusieurs jugements épistémiques dans lesquels l'évaluation des faits probants a été effectuée. Ce concept, avec sa référence à des jugements et des évaluations, est donc approprié pour la gnoséologie appliquée. Je définis par ailleurs le concept de croyance pour qu'il serve la gnoséologie pure, en tant qu'état mental involontaire d'un sujet. Les deux notions sont reliées, puisqu'exprimer une opinion consiste d'abord en la description d'un état mental (d'une croyance); à celle-ci doivent cependant s'ajouter l'affirmation et la défense rationnelle de son contenu pour que nous ayons une opinion dans le plein sens du terme. Par rapport à ces notions, la gnoséologie appliquée peut donc être caractérisée comme étant la discipline qui fait l'analyse des conditions sous lesquelles les croyances d'un sujet se transforment en opinions, par le moyen du passage à la conscience réflexive.

Le concept de fait probant est aussi utilisé de manière différente dans les deux projets gnoséologiques. Les faits probants sont dits " personnels "s'il sont impliqués dans une relation de support avec une opinion ou une croyance d'un sujet, selon l'optique de celui-ci ; il s'agit, en d'autres termes, des bonnes raisons épistémiques dont le sujet dispose de tenir ses croyances ou opinions pour vraies. Par exemple, le fait que l'éclairage soit normal et celui que mes yeux soient en bon état de fonctionnement sont deux faits probants personnels en faveur de mon opinion qu'il y a présentement un écran d'ordinateur en face de moi, car j'estime que tous deux supportent cette opinion. Les faits probants personnels se distinguent des faits probants objectifs, qui sont pour leur part impliqués dans des relations objectives avec d'autres faits : leur présence indique au moins une probabilité objective d'obtention d'un second fait. Par exemple, des traces de pas dans la neige indiquent que quelqu'un est passé par là. La relation entre les deux faits est qualifiée d'objective car elle existe indépendamment de toute évaluation de la situation que pourrait faire un sujet. La question des rapports entre faits probants personnels et faits probants objectifs est un problème délicat pour la gnoséologie appliquée : il peut être difficile, depuis la perspective limitée d'un sujet, de montrer que de bonnes raisons épistémiques de tenir une opinion pour vraie sont également des faits probants objectifs en sa faveur. Les raisons épistémiques qu'un sujet peut invoquer sont parfois entachées de préjugés ou d'erreurs de jugement, qu'il lui est difficile ou impossible d'entrevoir. D'autre part, ce ne sont pas toutes les formes de faits probants objectifs qui peuvent servir comme faits probants personnels puisque ces derniers doivent toujours être accessibles à la conscience du sujet. La gnoséologie pure, de son côté, peut utiliser sans contrainte l'une ou l'autre des notions. 
La distinction entre gnoséologie pure et gnoséologie appliquée ne doit pas être confondue avec certaines autres distinctions qui existent dans la littérature ${ }^{9}$. Je pense ici, par exemple, au contraste entre l'internalisme et l'externalisme. On pourrait ètre porté à croire que l'internalisme, qui est la thèse selon laquelle les faits probants doivent être accessibles à la conscience du sujet, correspond de ce fait à la gnoséologie appliquée. Il faut bien voir, cependant, que l'internalisme, puisqu'il concerne la nature des faits probants et non pas les évaluations des sujets à propos de ces faits, est une thèse en gnoséologie pure. D'ailleurs, les éléments du couple internalisme/ externalisme sont en opposition, tandis que ceux du couple gnoséologie pure/gnoséologie appliquêe sont complémentaires. La confusion possible est attribuable au fait que le sujet épistémique doit avoir un accès conscient aux faits probants pour ètre capable d'effectuer des évaluations. Méfions-nous aussi d'une assimilation trop hātive de la gnoséologie appliquée avec la connaissance de second degré : savoir que l'on sait n'équivaut pas, ou du moins pas toujours, à évaluer nos opinions. La meilleure façon de nous en rendre compte est de prendre un exemple, la théorie causale de la connaissance : si un sujet sait que $\mathrm{p}$ si et seulement si le fait que $\mathrm{p}$ cause la croyance que $p$, alors le sujet sait qu'il sait que $p$ si et seulement si le fait qu'il sait que $p$ cause sa croyance qu'il sait que $p$; or cette relation causale existe indépendamment de toute évaluation que pourrait entreprendre le sujet concemant le statut de son opinion qu'il sait que p.

Dire simplement qu'il doit y avoir une composante pure et une composante appliquée au sein d'une approche gnoséologique ne suffit pas pour se représenter adéquatement ma conception de celle-ci en tant que gnoséologie cartésienne. Cette dernière se caractérise surtout par une façon particulière de relier les deux composantes. La discussion suivante, qui porte sur les deux nouvelles contraintes méthodologiques que je propose de considérer, permettra de comprendre comment.

\section{Les contraintes d'objectivité et d'applicabilité}

J'espére en avoir assez dit sur la distinction entre les deux formes de gnoséologie qui constituent la gnoséologie cartésienne pour permettre la bonne compréhension des contraintes méthodologiques d'objectivité et d'applicabilité que je vais maintenant introduire. L'exigence d'objectivité peut toutefois sembler familière; les gnoséologues en parlent régulièrement en faisant référence aux standards épistémiques d'une théorie. Selon l'idée courante, une théorie a des standards épistémiques objectifs lorsque ceux-ci fonctionnent comme des filtres qui sélectionnent des croyances vraies (ou fausses, s'il

9. Elle semble toutefois correspondre au contraste entre méta-gnoséologie et gnoséologie normative discuté par Alan Goldman dans Empirical Knowledge, Berkeley, University of Califomia Press, 1988, p. 19-20. Par contre, je n'aime pas l'utilisation du préfixe "mèta * pour désigner la gnoséologie pure, car celle-ci n'est pas une entreprise méta-théorique sur la gnoséologie. Guant à la gnoséologie normative, elle met l'emphase, comme la gnoséologie appliquée, sur la perspective du sujet, mais je ne suis pas sûr que Goldman ne tende pas à la réduire parfois à l'application de la méta-gnoséologie (la gnoséologie pure) à des problèmes particuliers comme la perception. 
s'agit d'un standard négatif), indépendamment des idées du sujet sur la question ${ }^{10}$. Autrement dit, les seuls types de faits probants admissibles comme standards épistémiques au sein d'une telle theorie sont de nature objective, et non simplement personnelle. Toute croyance rencontrant les standards choisis a un certain taux de probabilité objective d'être vraie (ou fausse). Par exemple, la cohérence est un standard objectif négatif, car il y a obligatoirement au moins un énoncé faux dans un ensemble incohérent d'énoncés. Une croyance faisant partie d'un tel groupe a donc une certaine probabilité objective d'être fausse. En revanche, le caractère d'un standard comme l'inférence à la meilleure explication n'est pas aussi clair : pourquoi devrait-on penser que la meilleure explication d'un fait donné a une probabilité objective d'être vraie ? Cela dépend du type de spécifications que la théorie gnoséologique donne à la notion de meilleure explication. Si la meilleure explication du fait est en bout de ligne simplement celle qui semble ètre la meilleure au sujet, alors le standard est subjectif.

Je suis d'accord avec cette majorité de gnéosologues qui insistent pour que toute théorie gnoséologique acceptable ait des standards épistémiques objectifs. La gnoséologie ne doit pas perdre de vue que son objectif premier est la validation de nos croyances eu égard à la question épistémique de leur valeur de vérité, et non pas par rapport à leur caractère pratique ou éthique. Au contraire, selon moi l'exigence d'objectivité ne doit pas s'arrêter là. L'objectivité traditionnellement recherchée en gnoséologie se situe sur le plan de la gnoséologie pure - elle concerne la nature des conditions épistémiques elles-mêmes - tandis que je la recherche aussi au sein de la gnoséologie appliquée. À ce niveau, elle se traduit comme étant la condition que les sujets doivent procéder à l'évaluation de leurs opinions selon une perspective objective.

Mais qu'est-ce qu'une perspective objective, dans le contexte de la gnoséologie appliquée, et comment peut-on la distinguer de la " perspective " absolutiste de la gnoséologie pure ? Afin de répondre à ces questions, rappelons d'abord la position du gnoséologue pur: celui-ci ne fait aucune évaluation, se contentant de qualifier des situations données, dont il connaît l'entièreté parce qu'elles ne sont qu'hypothétiques, en fonction de certains concepts épistémiques. Bien sūr, cette position n'est jamais accessible au sujet pour la gestion de sa conduite épistémique, car celui-ci se trouve toujours dans des situations réelles. Comment peut-il donc faire, depuis son point de vue limité, pour obtenir une plus grande objectivité ? Comment peut-il, en d'autres termes, se donner une perspective objective pour effectuer l'évaluation de ses opinions? Ma suggestion est d'utiliser les résultats de la théorie gnoséologique pure qu'il considère la meilleure. Plus spécifiquement, pour savoir si une de ses opinions constitue une occurrence de connaissance (par exemple), le sujet doit chercher à déterminer si les conditions constitutives de la connaissance, telles que définies par la version de la gnoséologie pure qu'il favorise, sont réalisées dans cette

10. Voir par exemple Alvin Goldman, Epistemology and Cognition. Cambridge, Harvard University Press, 1986, p. 69-73. 
circonstance à propos de cette opinion. Cela équivaut à effectuer un jugement épistémique sur la présence de ces conditions, qui constituent la teneur des faits probants que le sujet doit rechercher. Dans ces circonstances, si les standards et les concepts épistémiques de la gnoséologie pure sont par ailleurs objectifs, la réalisation des conditions qu'ils spécifient servira de fait probant objectif en faveur de la croyance du sujet. Par exemple, un partisan du fiabilisme (reliabilism) - la thèse gnoséologique selon laquelle une croyance acquiert le titre de connaissance lorsqu'elle a été produite par un processus cognitif à haut rendement de vérité devrait procéder à la détermination du rendement du processus cognitif par lequel il a formé sa croyance. La présence des conditions spécifiées par le fiabilisme garantira alors, par définition, une probabilité objective que la croyance soit vraie. Évidemment, cela n'assure pas que le sujet soit infaillible dans son évaluation de la présence du standard. J'aj déjà reconnu que tout jugement épistémique comporte des risques d'erreur.

Contrastons l'idée de perspective objective avec différents types d'évaluations faites selon des perspectives subjectives. Cela pourrait d'abord être celles d'un sujet ne possédant qu'une connaissance intuitive du concept de connaissance, parce qu'il n'a pas accompli de réflexion gnoséologique sur la question. Cela pourrait aussi ètre deux cas qui concement davantage les gnoséologues - les évaluations d'un sujet ayant effectué une telle réflexion, mais qui, pour une raison ou pour une autre, n'en utilise pas correctement les résultats, présumément sans s'en rendre compte ; ou encore celles d'un sujet qui ne fait que se conformer aux standards épistemiques ou aux pratiques évaluatives prévalant dans sa communauté, sans véritablement en faire l'examen critique, du point de vue de leur relation avec la vérité. Dans tous ces cas le sujet ne procède pas à l'évaluation critique de ses opinions de façon optimale : soit qu'il prenne quelque chose pour acquis (que ses concepts épistémiques intuitifs ou ceux de sa communauté sont adéquats ou que les pratiques évaluatives de sa communauté sont valables), soit qu'il ne soit pas totalement cohérent dans sa démarche pour obtenir une plus grande objectivité.

L'empirisme de Bas van Fraassen constitue à mon avis un exemple de gnoséologie appliquée subjective, au sens où elle ne relève pas d'un examen critique de la relation entre le standard épistémique qu'elle favorise et la vérité. Selon van Fraassen, ètre un empiriste, c'est adopter la méthodologie scientifique comme paradigme de rationalité, ce qui revient, selon son interprétation de la science (van Fraassen n'est pas un réaliste à propos des théories qui postulent l'existence d'entités inobservables), à ne reconnaitre que l'expérience sensible comme source d'information acceptable à propos du monde. C'est dire, selon ma terminologie, que la provenance sensible d'une opinion factuelle est un type de fait probant personnel à rechercher pour son évaluation favorable. L'empirisme de van Fraassen n'est donc pas un énoncé de fait sur des limites de nos sources de connaissance, mais une contrainte méthodologique sur l'évaluation de la rationalité d'opinions factuelles. Cependant, pour fonder objectivement une telle contrainte normative sur la rationalité, c'est-à-dire pour que cette contrainte soit telle que 
sa satisfaction par certaines opinions garantisse leur vérité au moins selon une probabilité objective, il faudrait ou bien accepter l'énoncé de fait selon lequel les sens constituent la seule et unique source d'information à propos du monde, ou encore celui selon lequel la méthode scientifique a effectivement un haut rendement de vérité (au sens du fiabilismel pour l'obtention de ce type d'information; mais van Fraassen rejette explicitement ces deux énoncés ${ }^{11}$. En l'absence d'un tel fondement, cependant, la restriction empiriste sur les sources d'information acceptables demeure subjective. Cela n'implique pas qu'un sujet n'aurait pas de bonnes raisons épistémiques, selon sa perspective propre, de l'utiliser pour l'évaluation de ses opinions factuelles; cela veut seulement dire que sa relation avec la vérité n'a pas été démontrée. Le sujet épistémique ayant mené une réflexion gnoséologique doit, dans ces circonstances, reconnaitre qu'il n'a pas plus de raison objective d'adopter une méthodologie empiriste qu'une méthodologie rationaliste ou mystique. Son choix peut alors s'expliquer par des raisons sociales, comme par exemple par sa haute estime de la communautê scientifique, qu'il se représente comme étant essentiellement empiriste.

Ce manque d'objectivité au niveau de la gnoséologie appliquée se retrouve également au sein de l'empirisme de Peter Carruthers. Pour Carruthers, comme pour van Fraassen, l'empirisme n'est pas un énoncé factuel mais une contrainte normative, qui fait partie, cette fois, dune politique pour la justification des croyances. Sa gnoséologie pure est objective, basée sur l'idée, précédemment introduite, que la connaissance peut ètre définie en termes de rendement des processus cognitifs. Au niveau de la gnoséologie appliquée, Carruthers requiert en plus que nos croyances à propos de la fiabilité des processus impliqués ne soient pas en contradiction avec la conception que la science s'en fait. La science est donc selon lui la seule source de justification acceptable à leur propos, la seule méthode dont nous disposons pour en vérifier le rendement ${ }^{12}$. Cette forme d'empirisme au niveau de la gnoséologie appliquée n'est à mon avis pas objective, car elle n'est pas autorisée par la gnoséologie pure dont elle devrait ètre issue : alors que la gnoséologie pure de Carruthers permet qu'un sujet puisse évaluer ses opinions comme étant justifiees dès qu'il peut établir qu'elles ont été produites par un processus cognitif fiable, sa gnoséologie appliquée spécifie en plus que seule la science est en mesure de produire ce type de démonstration. Or, en l'absence de preuves de la capacité de la science et de l'incapacité de tous les autres processus cognitifs potentiels (comme par exemple l'intuition rationnelle en métaphysique et l'intuition mystique) à cet égard, la contrainte normative de Carruthers demeure subjective : elle ne fait que refléter son opinion personnelle, indépendamment de sa réflexion gnoséologique. Nous

11. Il s'agit bien ici de l'empirisme gnoséologique de van Fraassen, et non pas de son empirisme constructif en philosophie des sciences. Voir, par exemple, "Against Naturalized Empiricism", dans P. Leonardi \& M. Santambrogio, eds, On Quine, Cambridge. Cambridge University Press, 1995, et "Against Transcendental Empiricism ", dans T. G. Stapledon, ed., The Question of Hermeneutics, Dordrecht, Kluwer, 1994.

12. Human Knowledge and Human Nature, Oxford, Oxford University Press, 1992. 
verrons plus loin qu'il y a d'ailleurs de bonnes raisons de penser que de telles preuves de la validité de processus cognitifs généraux sont impossibles à obtenir dans le cadre d'une théorie comme le fiabilisme.

Dans le contexte qui vient d'être explicité, il est aisé de comprendre la nature de la contrainte méthodologique d'applicabilité : elle est simplement la condition que les concepts épistémiques de la théorie de gnoséologie pure ayant été sélectionnée soient applicables, c'est-à-dire tels que le sujet soit à mème de les utiliser pour procéder à l'évaluation (objective) de ses opinions. Nous aurions, dans le cas contraire, une situation où le sujet est forcé d'admettre que le statut épistémique de ses opinions est de nature métaphysique : il ne pourrait jamais y avoir de considération probante en faveur ou en défaveur de l'affirmation que telle opinion a tel statut. Comment, en effet, un sujet peut-il faire cette détermination s'il n'a jamais d'accès conscient aux conditions d'application des concepts épistémiques qu'il emploie?

Contrairement aux apparences, cette exigence d'applicabilité ne va pas de soi : il existe une famille renommée de théories gnoséologiques qui ne la respecte pas. À la suite des travaux d'Alvin Goldman, un secteur important de la gnoséologie est aujourd'hui consacré à l'articulation et à la défense de théories de la connaissance et de la justification basées sur l'idée de rendement mentionnée plus haut ${ }^{13}$. On peut vérifier l'applicabilité de ces théories en considérant par exemple une situation où un sujet doit déterminer le rendement du processus cognitif par lequel il acquiert ses croyances perceptuelles. Que peut-il faire? Une suggestion immédiate serait de mesurer la proportion d'énoncés vrais dans un échantillon représentatif de croyances obtenues par la perception et d'inférer qu'il s'agit là du rendement de ce processus cognitif. Le problème avec ce type de procédé, discuté en détail par William Alston, est la circularité : il faut, pour déterminer la fiabilité d'un processus cognitif, employer ce même processus. Utiliser des procédés plus élaborés, qui font appel à d'autres processus cognitifs (comme par exemple invoquer une inférence à la meilleure explication de la structure de nos expériences perceptuelles pour démontrer la fiabilité de la perception) ne résout pas le problème, puisqu'il faudra aussi, tôt ou tard, établir la propre validité de ces processus. Comme le dit Alston,

[...] si nous persistons suffisamment dans notre questionnement, nous allons ou bien (a) rencontrer un autre processus cognitif pour lequel une démonstration non circulaire ne peut ètre donnée, ou (b) donner une preuve circulaire, ou (c) nous retrouver dans une régression à l'infini (traduction personnelle) ${ }^{14}$.

La conclusion est que ce type de théorie n'est pas applicable ou, peut-être plus exactement, n'est applicable qu'au prix d'une circularité épistémique : pour qualifier une opinion de connaissance

13. Voir, par exemple, Goldman, op. cit. et Liaisons, Cambridge, MIT Press, 1991 .

14. William Alston, The Reliability of Sense Perception, Ithaca, Cornell University Press, 1993, p. 118. 
ou de croyance justifiée, il faut ègalement supposer la connaissance ou la justification d'opinions similaires. La connaissance demeure, dans le contexte d'une théorie comme le fiabilisme, une possibilité dont la réalité ne peut jamais être démontrêe par un sujet.

Il existe une autre famille de concepts épistêmiques souffrant de ce même problème de non-applicabilité. J'ai mentionné plus haut l'existence du problème de Gettier qui embarrasse la théorie de la connaissance traditionnelle : dans certaines situations où un sujet a une croyance vraie justifiée, l'intuition demeure pourtant celle qu'il ne sait pas. De nombreux gnoséologues ont réagi en proposant l'addition d'une quatrième condition à la théorie traditionnelle, en plus de celles de vérité, de croyance et de justification, qui consiste à préciser la nature de la justification devant ètre recherchée. Cependant, la structure de cette condition est souvent telle qu'elle n'a de valeur que conceptuellement, son seul but étant de lier entre elles les conditions constitutives de la justification et de la connaissance. La condition s'avère en pratique inutilisable pour un sujet s'intéressant à la validité de ses opinions. Considérons, par exemple, la version adoptée par Moser, qui fait appel à la notion de justification qui résiste, quant à sa vérité :

La justification $\mathrm{J}$ du sujet $\mathrm{S}$ pour la proposition $\mathrm{P}$ résiste, quant à sa vérité, si et seulement si il existe, pour chaque proposition vraie $\mathrm{V}$ qui, conjointement avec $\mathrm{J}$, annule la justification $\mathrm{J}$ de $\mathrm{S}$ pour $\mathrm{P}$, une proposition $\mathrm{V}$ qui, conjointement avec $\mathrm{J}$ et $\mathrm{V}$, restitue la justification $\mathrm{J}$ de $\mathrm{S}$ pour $\mathrm{P}$ de façon à ce que $S$ soit en réalité justifiè de croire en $\mathrm{P}^{15}$

Quelle recommandation pratique le sujet peut-il tirer de cette notion? Celle de considérer l'une après lautre toutes les possibilités logiques de remise en question de la justification dont il dispose, pour chaque opinion analysée. Il va de soi que l'application de cette procédure est problématique; et la situation ne s'améliore pas de façon très significative si l'examen est limité à la considération de toutes les possibilités permises par les lois naturelles, comme une autre théorie similaire discutée par Moser le suggère.

Pour ramener la tàche du sujet épistémique à des proportions plus maniables, dans le cadre de la théorie traditionnelle de la connaissance, il faudrait par exemple restreindre le champ d'investigation de la valeur de la justification aux seules possibilités d'erreur jugées pertinentes dans une situation donnée. Le problème est alors cependant de définir la notion de pertinence de façon à ce qu'elle ne soit pas purement subjective, car autrement la manœuvre suggérée n'aura pour effet que de déplacer le problème d'un lieu à un autre: d'une version non-applicable de la gnoséologie pure à une version subjective de la gnoséologie appliquée. Cet exemple est instructif car il montre à quel point le respect simultané des deux contraintes méthodologiques que je propose n'est pas trivial. S'il est

15. Voici la version originale anglaise de cette traduction personnelle, que l'on trouve dans Paul Moser, Knowledge and Evidence, Cambridge, Cambridge University Press, 1989 : "S's justifying evidence E for P is truth-resistant if and only if for every true proposition $T$ that, when conjoined with $E$, contravenes $S^{\prime}$ 'sustification for $P$ on $E$, there is a true proposition, $T^{\prime}$, that when conjoined with $\mathrm{E} \& \mathrm{~T}$, restores the justification of $\mathrm{P}$ for $\mathrm{S}$ in a way that $\mathrm{S}$ is actually justified in believing $P$ " (P. 245). 
facile de définir des standards subjectifs et applicables (pensez à n'importe quelle directive qu'il vous convient de respecter), et relativement aisé de produire des standards objectifs et non applicables (il suffit d'adopter la perspective absolutiste de la gnoséologie pure et de caractériser quelque condition prévalant indépendamment de ce que le sujet pense, sans poser la question de sa perspective réflexive), il en va autrement lorsqu'il s'agit de réaliser à la fois l'objectivité et l'applicabilité. La gnoséologie semble souvent osciller entre ces deux pōles, sans pouvoir trouver de position d'équilibre. Exiger de toute théorie gnoséologique qu'elle rencontre cette double exigence peut donc sérieusement réduire le champ des candidates acceptables, ce qui était le premier but recherché.

\section{L'ascension méta-gnoséologique}

À la conclusion de la section 3 , j'ai affirmé que la gnoséologie cartésienne constitue une façon particulière de relier la composante pure et la composante appliquée d'une théorie gnoséologique. Plus tôt dans cette même section, j'avais également mentionné que la gnoséologie cartésienne est pour moi la thèse selon laquelle la gnoséologie a pour but de donner des règles de conduites objectives. à un niveau très général, aux sujets épistémiques qui font l'évaluation du statut de leurs opinions dans le but d'acquérir un système ètendu de croyances vraies. Il est maintenant possible de comprendre comment ces deux affirmations se rejoignent. La définition parle de sujets qui procèdent à l'évaluation de leurs opinions par rapport à l'atteinte d'un but épistémique : toute gnoséologie qui veut réaliser cet objectif se doit donc d'avoir une composante appliqueee. La définition mentionne également que les règles de conduite utilisées à cet égard doivent être objectives ; j'ai suggéré ce qui m’apparait être la forme optimale d'objectivité à ce niveau, en tenant compte des limites de la perspective réelle d'un sujet: se tourner vers une gnoséologie pure dont les concepts et les standards sont objectifs, afin d'identifier les conditions à rechercher comme faits probants personnels. Or, c'est précisèment de cette façon que les composantes pure et appliquée sont liées au sein de la gnoséologie cartésienne. Cette dernière est donc simplement une version objective de gnoséologie appliquée basée sur une version objective de gnoséologie pure - une gnoséologie objective et applicable.

La discussion du paragraphe précédent révèle clairement que la gnoséologie cartésienne, telle que présentée dans cet article, est uniquement une thèse méta-gnosèologique - une thèse à propos de la gnoséologie en général, exprimant une préférence envers une certaine conception de celle-ci - et non une théorie gnoséologique spécifique. Le lecteur aura d'ailleurs peut-être trouvé que ce texte ne fait que dresser les grandes lignes d'un projet, sans être véritablement substantiel. Je dois cependant lui rappeler qu'il s'agit là du propre de toute discussion d'ordre méta-gnoséologique, tant qu'elle n'est pas complétée par la gnoséologie qu'elle annonce. Pour poursuivre la question dans le sens proprement gnoséologique, il faudrait chercher à déterminer des concepts et des standards épistémiques répondant aux deux conditions que j'ai posées. S'il est démontré que plusieurs concepts ou standards de ce type existent, 
cela signifie qu'on peut élaborer différentes versions de gnoséologie cartésienne. Je ne crois pas, cependant, que ces théories puissent être contradictoires, du moins si l'on admet qu'il n'y a qu'une seule vérité, parce qu'elles doivent toutes respecter la contrainte d'objectivité sur le plan de la gnoséologie pure. Il faut plutôt penser que les concepts et standards seraient complémentaires, agissant à différents niveaux de rigueur ou de discours.

Remarquons comment la réduction du nombre de théories gnoséologiques potentiellement acceptables se fait, dans cette approche basée sur les contraintes mèthodologiques, par l'intermédiaire d'un passage au niveau mèta-gnoséologique : ce ne sont pas directement les théories comme telles qui sont discréditées, mais les projets gnoséologiques desquels elles sont issues. J'ai par exemple remis en question tous les projets qui mettent de l'avant des standards ou des concepts épistémiques faisant référence de façon essentielle à la préférence des sujets et tous les projets qui se limitent à décrire les pratiques évaluatives habituelles d'un sujet ou d'une communauté, chaque fois pour raison de subjectivité (ces projets sont respectivement des versions subjectives de gnoséologie pure et de gnoséologie appliquée). Ont également été mises de côté toutes les versions de la gnoséologie qui se contentent de spécifier des conditions épistémiques en perdant de vue la perspective du sujet réflexif, parce que considérées comme non applicables ou incomplètes. Seul demeure le projet cartésien, tel que je l'ai défini. Ceci suggère que l'origine des désaccords profonds qui marquent la scène des discussions en gnoséologie contemporaine se situe probablement, en fait, sur le plan méta-gnoséologique, là où se conçoivent, de façon plus ou moins explicite, les différents projets gnoséologiques. La diversité autorisée quant à la nature de ces projets rejaillit au plan gnoséologique, se manifestant par un foisonnement de théories et concepts épistémiques souvent contradictoires.

Il est toutefois important de comprendre que les contraintes d'objectivité et d'applicabilité ne sont pas des artifices arbitraires que j'impose aux théories gnoséologiques dans le seul but d'en réduire le nombre. En réalité, elles suivent plutōt de ma conception du but de la gnoséologie, exprimée dans ma définition du projet cartésien. Une théorie subjective ou non applicable n'est pas acceptable pour moi, puisqu'elle ne peut assurer la double fonction que j'ai conférée à cette discipline : permettre d'évaluer et d'évaluer objectivement. Ces fonctions attribuées à la gnoséologie ne sont pas non plus arbitraires. Elles prennent origine dans mon intérèt, à titre de sujet épistémique, pour les croyances vraies accessibles. C'est pourquoi je ne considèrerais satisfaisante une approche rapportant les pratiques évaluatives de notre époque que si elles avaient èté validêes par rapport à un objectif de vérité - les préférences de chacun ou de la majorité ne m'intéressent pas dans la mesure où elles ne sont que cela: des préférences. Pas davantage satisfaisante à cet égard serait une théorie qui ne permet pas d'évaluer, car je cherche à accéder à la vérité : montrer que la connaissance est possible n'est peut-être pas trivial, mais je voudrais aussi savoir, pour autant que cela se peut, si cette connaissance possible est effectivement la mienne.

L'ascension méta-gnoséologique permet donc de situer la discussion sur le plan de la conception des divers projets 
gnoséologiques et des contraintes méthodologiques qu'ils imposent sur toute théorie gnoséologique satisfaisante. J'ai jusqu'à présent insistè sur les avantages d'un tel déplacement de la problématique ; il me faut maintenant en préciser les limites. Lorsqu'elle demeure sur le plan gnoséologique, la gnoséologie traditionnelle apparaît trop souvent comme une bataille d'intuitions attribuable au fait que les gnoséologues n'utilisent pas les mémes concepts épistémiques. Il n'est pas totalement inexact de dire de façon similaire que la gnoséologie traditionnelle devient, sur le plan méta-gnoséologique, une bataille de stipulations sur le sens à donner au terme "gnoséologie". Il serait donc tout à fait légitime pour un lecteur ayant parcouru ce texte, de rejeter la gnoséologie cartésienne, et par le fait même les contraintes d'objectivité et d'applicabilité, en faisant valoir qu'elle ne correspond pas à la gnoséologie telle qu'il se la représente; ou encore d'accepter ma proposition théorique comme une forme particulière de gnoséologie, à considérer en parallèle avec d'autres projets gnoséologiques, pourvu que ceux-ci soient poursuivis de façon cohérente, en accord avec le but et la méthode qu'ils se sont fixés. À ce niveau, mon argumentation ne peut que se limiter à réitêrer mon intêrêt premier pour l'évaluation objective des opinions, par rapport aux autres buts couramment recherchés; elle ne peut justifier un rejet total des autres formes de gnoséologie. Toutefois, cet intérêt, duquel je tire les contraintes méthodologiques qui font le travail d'êlimination, n'est pas évaluable ou critiquable gnoséologiquement, car même s'il vise un objectif épistémique, il n'est pas lui-même d'ordre épistémique. C'est pourquoi il ne saurait être question ici d'objecter que mon entreprise méta-gnoséologique échoue parce qu'elle tombe sous le coup de la circularité, ne réussissant pas à s'êlever au niveau de généralité désiré.

L'ascension méta-gnoséologique ne permet donc pas d'exclure une certaine variété, attribuable à la diversité des intérêts personnels des théoriciens, dans l'élaboration de projets gnoséologiques particuliers. Elle conserve tout de mème le mérite d'attirer l'attention sur l'importance qu'il y a, pour un auteur, à faire en sorte que les objectifs et la méthode de son projet philosophique soient clairement définis. Il pourra ainsi s'éviter d'attirer des critiques non pertinentes, et de s'en prendre à des rivaux qui n'en sont pas. Par exemple, certains fiabilistes reprochent à la théorie traditionnelle de la connaissance de faire appel à la notion de raison épistémique (justificative), invoquant le fait que des sujets peu sophistiqués peuvent connaitre sans toutefois ètre capables d'identifier les raisons qui justifient leurs croyances. Le partisan de la théorie traditionnelle réplique en insistant sur la nécessité d'inclure le concept de raison épistémique au sein de celui de connaissance pour exclure les cas où la chance fait en sorte que les croyances des sujets sont vraies. Toute cette discussion pourrait pourtant être évitée si les parties en présence réalisaient que la théorie traditionnelle de la connaissance mêlange des éléments de gnoséologie pure et des éléments de gnoséologie appliquée. Le partisan de la théorie traditionnelle a l'intuition que la perspective réflexive du sujet doit ètre prise en considération, comme en convient la gnoséologie appliquée, mais traduit à tort cette exigence en insérant la notion de raison épistémique comme condition constitutive du concept de connais- 
sance de sa gnoséologie pure. Le fiabiliste a raison de dire que connaitre et démontrer que l'on connait sont deux choses différentes, mais ceci ne suffit pas pour rejeter la notion de raison èpistémique hors du champ de la gnoséologie, à moins de réduire celle-ci à la gnoséologie pure. On voit bien ici que fiabilistes et traditionalistes ne se consacrent pas, en définitive, aux mèmes projets gnoséologiques ${ }^{16}$.

Remarquons que nous avons ici une explication possible de la grande variété des points de vue gnoséologiques autres que le relativisme - ce qui était le second objectif recherché dans cet article. J'avancerai donc la thèse que la diversité apparente au sein de la gnoséologie contemporaine est symptomatique du fait que ses fondements demeurent trop souvent dans l'ombre. La discussion n'a pas suffisamment porté, de façon systematique et explicite, sur la question méta-gnoséologique de la définition des différents projets gnoséologiques, avec le résultat que plusieurs projets distincts sont réunis sous une même bannière, ce qui tend fallacieusement à traduire de simples différences en oppositions. Dans cet article, j'ai tenté de montrer que la réhabilitation de la discipline dans son ensemble doit passer par un resserrement méthodologique qui permettra de distinguer et d'évaluer ces projets les uns par rapport aux autres. En ce sens, mon propos joue à deux niveaux : d'une part, je reconnais que des projets distincts peuvent très bien cohabiter au sein de la gnoséologie, au sens large du terme, pendant que j'affirme, d'autre part, qu'une forme particulière de projet gnoséologique devra ètre préférée.

Je suis maintenant en mesure de justifier le parti pris de ma démarche réductrice de la diversité d'approches et de théories gnoséologiques, affiché sans argumentation en introduction : dans le cadre du projet d'une gnoséologie cartésienne, et dans la mesure où il existe au moins une théorie gnoséologique de ce type, le relativisme ne constitue pas une option acceptable, car il exclut l'idée d'évaluation objective (si l'on considère par ailleurs qu'il n'y a qu'une vérité). C'est donc, en définitive, mon intérêt de théoricien qui fonde ma prise de position initiale.

\section{Pour une approche descendante des questions gnoséologiques}

L'approche des questions gnoséologiques que je favorise se caractérise en ce qu'elle est decendante : elle va du haut vers le bas, du plus théorique vers le moins théorique, en mettant l'accent sur les contraintes méthodologiques. Selon moi, l'analyse doit s'amorcer au niveau méta-gnoséologique, par l'identification précise du but et de la méthode d'un projet gnoséologique, pour ensuite se déplacer vers l'élaboration de théories qui rencontrent les exigences formulées, en autant qu'elles préservent nos intuitions dans les situations (vraiment) non conflictuelles. Même si l'approche descendante n'interdit pas de remettre en question certaines intuitions communes,

16. Á la question de savoir pour qui les attributions de haut rendement de vérité des processus cognitifs constituent des faits probants, Marshall Swain, un fiabiliste, répond dans Reasons and Knowledge (Ithaca and London, Cornell University Press, 1981) : "peut-être pour personne". (p. 101). On ne saurait trouver plus claire illustration de la différence des tâches entre la gnoséologie pure et la gnoséologie appliquée. 
il faut tout de mème que les concepts épistémiques courants soient reconnaissables dans la théorie gnoséologique, car ceux-ci sont déjà informès par les intérêts à visée épistémique à l'origine de la gnoséologie en tant que discipline philosophique. Proposer des concepts épistémiques sans aucun point de contact avec le sens commun équivaudrait selon toute probabilité à définir de nouveaux intérêts et, par le fait mēme, à changer de discipline ou de problèmatique.

Sur le plan méta-gnoséologique, j'ai proposé une version du traditionalisme, baptisèe " gnoséologie cartésienne", dont le but est l'élaboration de règles de conduite générales pour l'évaluation objective, par un sujet du statut épistèmique, de ses opinions, et dont la méthode consiste à spécifier des concepts qui rencontrent certaines exigences associées à ce but. Ma conception de la gnoséologie implique que celle-ci doit avoir une composante appliquée, en tant que complément à la composante pure, qui ne sert qu'à définir des concepts et des standards épistémiques. Elle implique aussi que tout concept épistémique de la composante pure doit être applicable, sous peine d'ètre rejeté comme n'étant d'aucun apport pratique pour la direction de la conduite épistémique du sujet. La motivation derrière la contrainte méthodologique d'objectivité est également directement liée à ma conception de la gnoséologie, en tant que discipline concernée non pas par la simple description des pratiques évaluatives courantes d'une communauté ou des préférences d'un sujet à cet égard, mais par la détermination des concepts et des standards épistemiques en fonction desquels l'on doit évaluer nos opinions si la vérité est notre but. Une gnoséologie objective et applicable contient donc des standards épistémiques objectifs, en fonction desquels des évaluations objectives peuvent ètre effectuées ; elle n'est pas pour autant exempte de toute subjectivité puisque celleci est incontournable dès qu'il est question d'évaluation ${ }^{17}$.

Mon approche méthodologique des questions gnoséologiques se distingue de celle, plus habituelle et ascendante, selon laquelle l'objectif premier de la démarche est la rationalisation d'intuitions personnelles, parfois contestables, par la construction de théories qui les supportent (je pense par exemple à tous ceux qui affirment que la gnoséologie se doit de réfuter le sceptique, parce qu'ils sont convaincus d'avance que celui-ci a tort ${ }^{18}$ ), et dans laquelle la dimension méta-gnoséologique du problème n'est trop souvent abordée que plus tard, comme une question d'importance secondaire. Je crois que l'application de cette methode courante est en bonne

17. Ce que j'appelle le problème de la profondeur du jugement épistèmique - la question de savoir à quel moment un sujet peut affirmer que sa recherche des faits probants personnels est suffisante - constitue un programme de recherche intéressant pour la gnoséologie appliquée. Le problème est abordé notamment par Stanley Clarke, dans Rational Acceptance and Purpose, Totowa, Rowman \& Littlefield, 1989, qui met en évidence la dépendance pragmatique du contexte à ce niveau. On pourrait se demander s'il est possible, ici aussi, de définir des conditions objectives qui viendraient réduire encore la subjectivité inhérente à toute démarche gnoséologique qui considère la perspective limitée du sujet.

18. Voir, par exemple, John Pollock. Contemporary Theories of Knowledge. Totowa, Rowman \& Littlefield, 1986, p. 3-7. 
partie responsable de la situation inconfortable dans laquelle se trouve la gnoséologie contemporaine et que mon approche peut contribuer de façon favorable à l'émergence d'un consensus plus grand dans cette discipline. Il demeure possible de critiquer les contraintes méthodologiques proposées, ou les applications particulières que j'en ai données, mais j'estime néanmoins avoir suggéré, en déplaçant la question au niveau méta-gnoséologique, au moins lamorce d'une procédure utilisable pour solutionner les problèmes de la gnoséologie traditionnelle.

Département de philosophie

Université du Québec à Montréal 